\title{
Correction to: a-Synuclein's Uniquely Long Amphipathic Helix Enhances its Membrane Binding and Remodeling Capacity
}

\author{
Anthony R. Braun ${ }^{1}$ (D) Michael M. Lacy ${ }^{3} \cdot$ Vanessa C. Ducas $^{3} \cdot$ Zhiming Chen $^{4} \cdot$ Tobias Baumgart $^{4}$. \\ Elizabeth Rhoades ${ }^{3}$. Jonathan N. Sachs ${ }^{2}$
}

Published online: 27 July 2018

๑) Springer Science+Business Media, LLC, part of Springer Nature 2018

\section{Correction to: \\ The Journal of Membrane Biology (2017) 250:183-193 \\ https://doi.org/10.1007/s00232-017-9946-1}

The original version of the article unfortunately contained error in author group; two authors were not submitted and published in the original version. Also the funding information is erroneously omitted.

Dr. Zhiming Chen and Dr. Tobias Baumgart are included as fourth and fifth authors in the author group.

The funder acknowledgement is given below:
The original article can be found online at https://doi.org/10.1007/ s00232-017-9946-1.

\section{Jonathan N. Sachs}

jnsachs@umn.edu

1 Department of Neuroscience, University of Minnesota, Minneapolis, MN 55455, USA

2 Department of Biomedical Engineering, University of Minnesota, Minneapolis, MN 55455, USA

3 Department of Molecular Biophysics and Biochemistry, Yale University, New Haven, CT 06520, USA

4 Department of Chemistry, University of Pennsylvania, Philadelphia, PA 19139, USA
Acknowledgements All simulations and analysis were completed at the Minnesota Supercomputing Institute (MSI).

Author Disclosures This work was supported in part by the National Institutes of Health R01 NS084998 (to JNS) and NRSA Fellowship F31 NS077634 (to ARB), in addition to GM102815 (to ER), predoctoral training grant GM008283 (to VCD and MML) and R01 GM 097552 (to ZC and TB). No competing financial interests have been declared. 